\title{
APTIDÃO CARDIORRESPIRATÓRIA, COMPOSIÇÃO CORPORAL DE ESCOLARES E ESTRUTURAS FÍSICAS PARA PRÁTICA DE ATIVIDADES FÍSICAS
}

\author{
CARDIORESPIRATORY FITNESS, BODY COMPOSITION OF \\ SCHOOLCHILDREN AND PHYSICAL STRUCTURE FOR PRACTICE \\ OF PHYSICAL ACTIVITIES
}

\begin{abstract}
Willian Giovani de Araújo', Silvana Silveira Soares², Ana Paula Sehn', Deise Graziela Kern', Letícia Welser', Cristiane Fernanda da Silva', Miria Suzana Burgos ${ }^{3}$, Cézane Priscila Reuter ${ }^{4}$
\end{abstract}

\section{RESUMO}

O presente estudo transversal objetiva comparar o nível de aptidão cardiorrespiratória (APCR) e a composição corporal de 393 escolares, pertencentes a escolas com e sem estrutura física no entorno para a prática de atividades físicas e esportivas em Santa Cruz do Sul - RS. Os níveis de APCR de escolares, tanto do sexo masculino, quanto do feminino, são superiores no ambiente que possui maior quantidade de estruturas esportivas em seu entorno. Não foi observada relação entre a infraestrutura e a composição corporal dos escolares. Conclui-se que escolares, de ambos os sexos, que estudam em escolas com estruturas esportivas em seu entorno, apresentam maiores níveis de APCR, em comparação aos escolares pertencentes às escolas com baixa presença de estruturas esportivas.

Descritores: Aptidão Física; Aptidão Cardiorrespiratória; Composição Corporal; Saúde Escolar.

\section{ABSTRACT}

This cross-sectional study aims to compare the level of cardiorespiratory fitness (CRF) and the body composition of 393 school children, with and without physical structure in the school environment for physical and sports activities in Santa Cruz do Sul - RS. Regarding CRF levels, it is observed that schoolchildren, both male and female, who study in study in schools with presence of sports structures in their environment have better values. There was no relation between infrastructure and body composition in school children. It's concluded that schoolchildren of both sexes who study in schools with sports structures in their surroundings present higher levels of CRF compared to schoolchildren belonging to schools with a low presence of sports structures.

Descriptors: Physical Fitness; Cardiorespiratory Fitness; Body Composition; School Health.
${ }^{1}$ Graduado(a) em Educação Física pela Universidade de Santa Cruz do Sul (UNISC), Santa Cruz do Sul, RS, Brasil.

${ }^{2}$ Acadêmica de Farmácia na Universidade de Santa Cruz do Sul (UNISC), Santa Cruz do Sul, RS, Brasil.

${ }^{3}$ Doutora em Ciências da Motricidade Humana pela Universidade Técnica de Lisboa (UTL), Lisboa, POR, Portugal.

${ }^{4}$ Mestre em Promoção da Saúde pela Universidade de Santa Cruz do Sul (UNISC), Santa Cruz do Sul, RS, Brasil. 


\section{Introdução}

A prática de atividade física na infância eadolescênciaé importante para prevençãoe e tratamento de algumas doenças crônicas, sendo que atividades classificadas como leves, moderadas ou intensas impactam na saúde cardiometabólica do indivíduo. Outros benefícios obtidos incluem melhorias na cognição, aptidão motora, função cerebral, no desempenho escolar e, ainda, o exercício acarreta em benefícios psicológicos, como melhora da autoestima e desenvolvimento de relações interpessoais ${ }^{1}$.

Níveis de aptidão cardiorrespiratória e de resistência física, assim como relação altura/peso, são importantes preditores de risco cardiometabólico, estando relacionados com a saúde global do escolar, assim como com sua auto percepção de saúde e sensação de bem-estar ${ }^{1,2}$. Por consequência, políticas de promoção de atividades físicas, que atuem na melhoria desses fatores, beneficiam o estado geral de saúde do jovem e também influenciam no estado de saúde e hábitos de estilo de vida desse indivíduo enquanto adulto ${ }^{3,4}$.

O ambiente social, econômico e físico em que o jovem está inserido também influi sobre esses fatores, podendo afetar positivamente ou negativamente os hábitos de vida do escolar de acordo com suas características e estruturas. Assim sendo, ambientes tidos como obesogênicos inferem em estilo de vida não saudável, geralmente desestimulando e inibindo a prática de atividade física, enquanto ambientes saudáveis estão geralmente associados a bons hábitos alimentares e estímulos às práticas de atividades físicas ${ }^{5}$.

Nesse contexto, o ambiente físico atua podendo encorajar a atividade conforme sua capacidade de estrutura ${ }^{6}$. Lugares que ofereçam uma gama de ambientes recreacionais e amplo acesso a áreas verdes ou estruturadas específicas para algum tipo de atividade ou esporte favorecem tais práticas. Ambientes que forneçam acessibilidade e possibilidade de transporte ativo seguro até chegar ao local da atividade também estão, em teoria, relacionados à propensão individual de manter uma vida mais ativa ${ }^{7,8}$.

Para crianças e adolescentes em idade escolar, a escola e seus arredores são um importante local de promoção de hábitos saudáveis e a qualidade de suas estruturas físicas e instalações é fundamental para seu uso $0^{9}$. As opções de estruturas que os arredores escolares oferecem para realização de atividades físicas e práticas esportivas, de quadras esportivas à playgrounds e parques verdes, promovem equidade de acesso aos alunos a um ambiente propício e seguro para essas práticas sem custos financeiros adicionais, mesmo naquelas atividades orientadas de programas em turno inverso ao escolar ${ }^{1,10}$.

Desse modo, o presente estudo tem como objetivo comparar o nível de aptidão cardiorrespiratória e a composição corporal de escolares pertencentes a escolas com e sem estrutura física em seu entorno, para a prática de atividades físicas e esportivas.

\section{Metodologia}

São sujeitos do estudo transversal 393 crianças e adolescentes (7 a 17 anos), de ambos os sexos, da zona urbana de Santa Cruz do Sul-RS. Foram avaliadas seis escolas do município, sendo que três delas apresentam estrutura para a prática de atividades físicas e esportivas no seu entorno e as outras três não possuem. As escolas 1, 2 e 3 possuem em seu entorno o campus da Universidade de Santa Cruz do Sul com campos de futebol 7 e 11, pista atlética, piscina coberta, quadras de esporte poliesportivas e ginásio poliesportivo; Parque da Oktoberfest com campo de futebol 11, pista de skate, pista de bicicross, canchas de bocha e eisstocksport, quadras de esportes poliesportivas e ginásio poliesportivo; campo de futebol 11 do Esporte Clube Avenida, além de praças com quadras brinquedos e aparelhos de ginástica. As escolas 4, 5 e 6 não possuem estrutura física para a prática de atividades físicas, esportivas e recreativas.

A frequência de prática de atividade física foi avaliada através de questionário autorreferenciado adaptado de Barros e Nahas ${ }^{11,12}$. Para avaliação da aptidão cardiorrespiratória (APCR), foi aplicado o teste de corrida/caminhada de seis minutos ${ }^{13}$. Previamente à coleta de dados, o escolar foi orientado a calçar tênis e vestir roupas leves. 0 teste foi aplicado na pista atlética da Universidade de Santa Cruz do Sul, previamente demarcada em metros. A distância percorrida pelo escolar foi posteriormente classificada em duas categorias: 1) normal (bons níveis de aptidão); 2) indicador de risco (baixos níveis de aptidão), de acordo com os pontos de corte estabelecidos pela literatura utilizada ${ }^{13}$. 
O estado nutricional do escolar foi avaliado pelo IMC, circunferência da cintura (CC) e percentual de gordura corporal (\%G). O IMC foi calculado pela fórmula IMC=peso/(estatura) $)^{2}$. Os valores contínuos foram classificados de acordo com os pontos de corte estabelecidos pela Organização Mundial da Saúde, em quatro categorias: baixo peso, normal, sobrepeso e obesidade. Para fins estatísticos, foram agrupadas as categorias baixo peso/normal, bem como sobrepeso/obesidade ${ }^{14}$. A CC foi medida com o escolar em pé e com braços estendidos. A avaliação foi realizada na parte mais estreita do tronco entre as costelas e a crista ilíaca. Os dados obtidos, em centímetros, foram posteriormente classificados, conforme os pontos de corte estabelecidos em duas categorias: normal e obesidade abdominal ${ }^{15}$. $\mathrm{O} \% \mathrm{G}$ foi avaliado, utilizando as dobras cutâneas tricipital e subescapular. Para o cálculo, foi utilizada a equação de Slaughter e colaboradores ${ }^{16}$. A classificação do $\% G$ foi realizada por meio dos pontos de corte estabelecidos em cinco classes: muito baixo, baixo, ótimo, moderadamente alto, alto e muito alto ${ }^{17}$. Posteriormente, os dados foram reclassificados em três categorias: muito baixo/baixo, ótimo e moderadamente alto/alto/muito alto.

Foi empregada a estatística descritiva para caracterização dos sujeitos, através dos valores de frequência absoluta e relativa, para as variáveis qualitativas, bem como média e desvio-padrão para as variáveis quantitativas. A normalidade dos dados contínuos foi previamente testada pelo teste de Shapiro-Wilk. A comparação entre as escolas com e sem estrutura física no seu entorno para a prática de atividades físicas, APCR e a composição corporal do escolar foi realizada, através do teste de qui-quadrado (variáveis qualitativas) e teste t de Student (variáveis quantitativas). Todas as análises foram realizadas no programa estatístico SPSS v. 23.0 (IBM, Armonk, NY, EUA), considerando significativas as diferenças para $p<0,05$.

\section{Resultados}

A tabela 1 apresenta as características descritivas dos escolares avaliados. Ressalta-se que $50,9 \%$ estudam em escolas com baixa presença de estruturas esportivas em seu entorno (Tabela 1).

Tabela 1 - Características descritivas dos escolares

\begin{tabular}{c|c}
\hline \multicolumn{2}{|c}{ Sexo } \\
\hline Masculino \\
\hline Feminino \\
\hline Faixa etária & $164(41,7)$ \\
\hline Criança (6-9 anos) & $229(58,3)$ \\
\hline Adolescente (10-17 anos) & $98(24,9)$ \\
\hline \multicolumn{2}{c}{ Presença de estrutura esportiva no entorno } \\
\hline Alta & $295(75,1)$ \\
\hline Baixa & $193(49,1)$ \\
\hline
\end{tabular}

A comparação dos valores médios das variáveis antropométricas e de APCR, de acordo com a presença de estruturas esportivas próximas às escolas avaliadas, demonstra que os níveis de APCR de escolares, tanto do sexo masculino, quanto do feminino, são superiores no ambiente que possui maior quantidade de estruturas esportivas em seu entorno. Para as variáveis antropométricas, não foram observadas diferenças significativas (Tabela 2). 
Tabela 2 - Comparação dos valores médios do estado nutricional e aptidão cardiorrespiratória, de escolas com e sem estrutura em seu entorno

\begin{tabular}{|c|c|c|c|}
\hline & \multicolumn{2}{|c|}{$\begin{array}{c}\text { Presença de estruturas esportivas } \\
\text { no entorno da escola }\end{array}$} & \multirow{3}{*}{$\mathrm{P}$} \\
\hline & Alta & Baixa & \\
\hline & Média (DP) & Média (DP) & \\
\hline \multicolumn{4}{|l|}{ Masculino } \\
\hline $\mathrm{IMC}\left(\mathrm{kg} / \mathrm{m}^{2}\right)$ & $19,76(3,73)$ & $19,98(4,04)$ & 0,723 \\
\hline $\mathrm{CC}(\mathrm{cm})$ & $66,69(9,82)$ & $67,38(11,11)$ & 0,673 \\
\hline Gordura corporal (\%) & $16,82(7,16)$ & $17,98(8,01)$ & 0,327 \\
\hline APCR $(m)$ & $988,94(202,36)$ & $927,90(191,27)$ & 0,049 \\
\hline \multicolumn{4}{|l|}{ Feminino } \\
\hline IMC (kg/m²) & $20,17(3,63)$ & $21,08(4,84)$ & 0,111 \\
\hline $\mathrm{CC}(\mathrm{cm})$ & $66,04(9,18)$ & $66,51(10,26)$ & 0,716 \\
\hline Gordura corporal (\%) & $21,95(5,72)$ & $23,41(6,28)$ & 0,068 \\
\hline $\mathrm{APCR}(\mathrm{m})$ & $829,34(131,76)$ & $797,31(108,84)$ & 0,048 \\
\hline
\end{tabular}

IMC: Índice de Massa Corporal; CC: Circunferência da Cintura; APCR: Aptidão cardiorrespiratória

Quando avaliada a classificação do estado nutricional e dos níveis de APCR (Tabela 3), observa-se que a frequência de escolares na zona de risco à saúde para a APCR é superior entre os escolares que estudam em escolas com baixa presença de estruturas esportivas no seu entorno, tanto entre os meninos $(p=0,015)$, quanto nas meninas $(p=0,006)$.

Tabela 3 - Comparação da classificação do estado nutricional e dos níveis de aptidão cardiorrespiratória, de escolas com e sem estrutura em seu entorno

\begin{tabular}{|c|c|c|c|}
\hline & \multicolumn{2}{|c|}{$\begin{array}{c}\text { Presença de estruturas esportivas } \\
\text { no entorno da escola }\end{array}$} & \multirow{3}{*}{$P$} \\
\hline & Alta & Baixa & \\
\hline & $\mathrm{n}(\%)$ & $n(\%)$ & \\
\hline \multicolumn{4}{|l|}{ Masculino } \\
\hline \multicolumn{4}{|l|}{ IMC } \\
\hline Baixo peso/Normal & $58(67,4)$ & $55(70,5)$ & \multirow{2}{*}{0,400} \\
\hline Sobrepeso/Obesidade & $28(32,6)$ & $23(29,5)$ & \\
\hline \multicolumn{4}{|l|}{ CC } \\
\hline Normal & $67(77,9)$ & $61(78,2)$ & \multirow{2}{*}{0,557} \\
\hline Elevada & $19(22,1)$ & $17(21,8)$ & \\
\hline \multicolumn{4}{|l|}{ Gordura corporal } \\
\hline Muito baixa/baixa & $23(26,7)$ & $11(14,1)$ & \multirow{3}{*}{0,083} \\
\hline Ótima & $36(41,9)$ & $44(56,4)$ & \\
\hline Mod. Alta/alta/muito alta & $27(31,4)$ & $23(29,5)$ & \\
\hline \multicolumn{4}{|l|}{ APCR } \\
\hline Zona saudável & $52(60,5)$ & $33(42,3)$ & \multirow{2}{*}{0,015} \\
\hline Zona de risco à saúde & $34(39,5)$ & $45(57,7)$ & \\
\hline
\end{tabular}




\begin{tabular}{|c|c|c|c|}
\hline & \multicolumn{2}{|c|}{$\begin{array}{c}\text { Presença de estruturas esportivas } \\
\text { no entorno da escola }\end{array}$} & \multirow{3}{*}{$\mathrm{P}$} \\
\hline & Alta & Baixa & \\
\hline & $n(\%)$ & $n(\%)$ & \\
\hline \multicolumn{4}{|l|}{ Feminino } \\
\hline \multicolumn{4}{|l|}{ IMC } \\
\hline Baixo peso/Normal & $69(64,5)$ & $78(63,9)$ & 0,521 \\
\hline Sobrepeso/Obesidade & $38(35,5)$ & $44(36,1)$ & \\
\hline \multicolumn{4}{|l|}{ CC } \\
\hline Normal & $78(72,9)$ & $96(78,7)$ & 0,192 \\
\hline Elevada & $29(27,1)$ & $26(21,3)$ & \\
\hline \multicolumn{4}{|l|}{ Gordura corporal } \\
\hline Muito baixa/baixa & $37(19,2)$ & $20(10,0)$ & 0,333 \\
\hline Ótima & $96(49,7)$ & $114(57,0)$ & \\
\hline Mod. Alta/alta/muito alta & $60(31,1)$ & $66(33,0)$ & \\
\hline \multicolumn{4}{|l|}{ APCR } \\
\hline Zona saudável & $42(39,3)$ & $28(23,0)$ & 0,006 \\
\hline Zona de risco à saúde & $65(60,7)$ & $94(77,0)$ & \\
\hline
\end{tabular}

IMC: Índice de Massa Corporal; CC: Circunferência da Cintura; APCR: Aptidão cardiorrespiratória

\section{Discussão}

Os resultados encontrados neste estudo, permitem constatar que para escolares de ambos os sexos a maior disponibilidade de estruturas esportivas próximas as dependências da escola acarreta em maiores níveis de APCR aos estudantes pertencentes a tais ambientes em comparação aos escolares que estudam em escolas que possuem pouca disponibilidade de estruturas esportivas próximas as suas dependências. Embora não se tenha encontrado neste estudo associação entre o estado nutricional e ambientes no entorno da escola. Os achados do presente estudo apontam importantes perspectivas relacionadas a ofertas de ambientes saúdaveis próximos aos entornos escolares. Um estudo realizado no Canadá encontrou estimativas semelhantes, sendo que maiores valores de APCR foram apresentados por escolares que passavam o tempo inverso ao turno escolar, em atividades ativas, na rua ${ }^{18}$. Essas estimativas também têm sido encontradas em populações mais velhas. No Texas - EUA, um estudo com adultos encontrou associação entre APCR e IMC e maior disponibilidade de locais para prática privada de exercício, assim como com a disponilidade de um número maior de áreas com ampla vegetação na vizinhança e APCR ${ }^{19}$. Do mesmo modo, um estudo no Taiwan, com uma amostra de adolescentes, encontrou melhores níveis de APCR, tanto para meninos como para meninas que dispunham de ambientes escolares com acesso a campo, quadras de esporte e ginásios ${ }^{20}$.

Estudos similares têm demonstrado efeitos positivos parecidos quanto a disponibilidades de ambientes favoráveis. Em estudo realizado no sudeste dos EUA, a associação entre acesso a parques e playgrounds e o IMC de meninos e meninas americanos foi moderada de acordo com o status socioeconômico, assim como pela etnia do escolar. Uma grande variedade de parques disponíveis está associada a valores menores de IMC em meninos com menor nível socioeconômico, mas com valores maiores de IMC, em meninos com maior nível socioeconômico. Já, para as meninas, alta disponibilidade de acesso a parques e playgrounds está associada a menor IMC para caucasianas com alto nível socioeconômico e com maior IMC, para afro americanas com baixo poder socioeconômico ${ }^{21}$.

Em estudo com garotas e garotos de origem hispânica e residentes em Los Angeles - EUA, encontrou-se associação entre a porcentagem de gordura corporal e o acesso a parques recreacionais somente para as meninas, sendo que aquelas que têm acesso fácil a mais de três parques na vizinhança tem uma redução de 1,4\% de gordura 
corporal quando comparadas aquelas que não têm acesso. Apesar de também terem tentado relacionar IMC e CC ao ambiente, os autores deste mesmo estudo mencionaram que, talvez o método utilizado em seu estudo, seja significativo somente ao avaliar medidas mais objetivas e com maior acurácia, como foi o caso da gordura corporal22.

Assim, aspectos relacionados aos ambientes escolares, mesmo que sem associação neste estudo, também acabam envolvendo as vivências alimentares. Um estudo com imigrantes nos Estados Unidos, relatou que o fácil acesso à comida saudável e a locais para atividades físicas está associado a menores valores de CC para imigrantes hispânicos, embora essa associação não reflita mudanças na CC com o passar do tempo ${ }^{23}$. Por sua vez, em crianças de Liverpool encontrou-se que o IMC e a CC são inversamente associados ao nível da vizinhança, sendo que não encontraram associação entre a vizinhança e APCR neste relato ${ }^{24}$.

Contudo, em nosso estudo, somente se avaliou o ambiente físico no entorno escolar. Entretanto, há evidências de que fatores sociodemográficos, principalmente o quesito social do ambiente, devem ser considerados como mais determinantes do que a estrutura física, ao promover um estilo de vida mais ativo e saudável25. Alguns autores afirmam que 0 status socioeconômico do estudante afeta seu uso das estruturas físicas na escola, enquanto que outros relatam que o nível socioeconômico da vizinhança do local da atividade física influencia mais no aproveitamento de suas estruturas físicas do que o nível socioeconômico do aluno ${ }^{26,27}$. Ainda, se sabe que a importância do suporte escolar para a prática física é mais relevante em países desenvolvidos do que em sub ou em desenvolvimento, assim como os melhores ambientes e infraestruturas se encontram nos países europeus e norte americanos, enquanto que os piores estão em países africanos ${ }^{28}$. Também, a auto percepção, ou a percepção dos pais ou responsável no caso de crianças e adolescentes, de segurança no local da atividade está entre os principais pontos de intervenção em política de incentivo à atividade física ${ }^{29,30}$.

Assim como, a qualidade da infraestrutura, e não somente sua quantidade, é determinante para a prática de atividade física. Em artigo sobre o nível de atividade física de crianças e adolescentes da África do Sul, cita-se que $81 \%$ das principais escolas de Western Cape precisavam de adequação e modernização em suas estruturas de práticas físicas, ainda que essas escolas fossem mais equipadas do que as de outras comunidades e, por isso, nem sempre 0 ambiente escolar propicia melhoras no estilo de vida ${ }^{31}$. Assim sendo, neste estudo, ter mais opções de práticas de atividades físicas ou não, não é um fator preditor significativo no estado nutricional de crianças e adolescentes. Portanto, encontrou-se que o estado nutricional da população avaliada independe da estrutura do seu ambiente escolar.

Escolares que estudam em escolas com e sem estruturas físicas para a prática de atividades físicas não demonstraram diferenças significativas nos índices de sobrepeso/obesidade, CC, gordura corporal ou IMC no presente estudo. Entretanto, um estudo realizado em Londres - UK, constatou que o ambiente do entorno escolar teve um modesto, porém significante, efeito sobre o IMC dos escolares ${ }^{32}$. Já, em outra pesquisa, com crianças e adolescentes de 5 a 18 anos da Pensilvânia, encontrou-se associação entre maior diversidade de locais para prática de atividades físicas e menor IMC, embora tenham encontrado diferenças quanto à significância de acordo com a idade ${ }^{33}$. Ainda, evidências de uma pesquisa recente, realizada com escolares portugueses, apontaram relação entre as estruturas físicas da escola e o nível de atividade física dos escolares, mas não com o IMC deles ${ }^{34}$.

Como limitações do presente estudo, não se analisou nível socioeconômico, mas somente o aspecto físico do ambiente utilizado, o que, como se identificou em outros estudos previamente citados, pode ter influenciado sobre 0 resultado aqui encontrado. Também, se fez análise quantitativa, mas não qualitativa das estruturas físicas, de modo que a acessibilidade e a disponibilidade do local para prática de atividade física não foram investigadas. Pelo fato de este estudo ser do tipo transversal, não se pode estabelecer qual a relação causal, que culminou na falta de relação entre a composição corporal e o uso das infraestruturas dispostas no entorno escolar. Contudo, os resultados encontrados e expostos no presente estudo, permitem delinear características primárias importantes de um contexto que precisa ser mais explorado, visto sua influência sobre o comportamento de saúde do escolares.

\section{Considerações Finais}

O presente estudo demonstrou que escolares de ambos os sexos que estudam em escolas com elevada presença de estruturas esportivas em seu entorno apresentam maiores níveis de aptidão cardiorrespiratória em comparação aos escolares pertencentes às escolas, com baixa presença de estruturas esportivas. Para o estado nutricional, não foram observadas diferenças significativas. 


\section{Referências}

1. Bangsbo J, Krustrup P, Duda J, Hillman C, Andersen LB, Weiss M, J. et al. The Copenhagen Consensus Conference 2016: children, youth, and physical activity in schools and during leisure time. BMJ Open Sport Exerc Med. 2016;50(19):1177-1178. 2. Kantomaa MT, Tammelin T, Ebeling H, Stamatakis E, Taanila A, et al. High levels of physical activity and cardiorespiratory fitness are associated with good self-rated health in adolescents. J Phys Act Health, 2015;12( 2): 266-272.

3. Dobbins M, Husson H, DeCorby K, LaRocca RL. School-based physical activity programs for promoting physical activity and fitness in children and adolescents aged 6 to 18. Cochrane Database Syst Rev, 2013; 2(2).

4. Ferro DA, Deijen JB, Koppes LL, van Mechelen W, Twisk JW, Drent ML. et al. The effects of physical activity and fitness in adolescence on cognition in adulthood and the role of insulin-like growth factor I. J Phys Act Health, 2016; 13(4): $392-402$.

5. Carroll-Scott A, Gilstad-Haydena K, Rosenthala L, Petersa SM, McCaslinb C, Joycea R, et al. Disentangling neighborhood contextual associations with child body mass index, diet, and physical activity: the role of built, socioeconomic, and social environments. Soc Sci Med. 2013; 95:106-114.

6. Bezold CP, Stark JH, Rundle A, Konty K, Day SE, Quinn J, et al. Relationship between recreational resources in the school neighborhood and changes in fitness in New York City public school students. J Urban Health. 2017: 1-10.

7. Loon JV, Frank L. Urban form relationships with youth physical activity: implications for research and practice. CPL bibliography. 2011; 26(3): 280-308.

8. Townshend T, Lake A. Obesogenic environments: current evidence of the built and food environments. Perspect Public Health. 2017; 137(1): 38-44.

9. Harrison F, Jones AP. A framework for understanding school based physical environmental influences on childhood obesity. Health Place. 2012; 18(3): 639-648.

10. Carlton TA, Kanters MA, Bocarro JN, Floyd MF , Edwards MB. et al. Shared use agreements and leisure time physical activity in North Carolina public schools. Am J Prev Med. 2017; 95S: S10-S16.

11. Barros MVG, Nahas MV. Medidas da atividade física: teoria e aplicação em diversos grupos populacionais. Londrina: Midiograf, 2003.

12. BURGOS MS. (Coord.). Saúde dos escolares - Fase III. Avaliação de indicadores bioquímicos, genéticos, hematológicos, imunológicos, posturais, somatomotores, saúde bucal, fatores de risco às doenças cardiovasculares e estilo de vida de escolares: estudo em Santa Cruz do Sul-RS (Projeto de Pesquisa). Santa Cruz do Sul: UNISC, 2015.

13. Projeto Esporte Brasil. Manual 2015 [online]. [Acesso em: 22 ago. 2016]. Disponível em: http://www.proesp.ufrgs.br.

14. World Health Organization. WHO 2007. Growth reference data for 5-19 years. 2007[online]. [Acesso em: 22 ago. 2016]. Disponível em: http://www.who.int/growthref/en/.

15. FERNÁNDEZ, J. R. et al. Waist circumference percentiles in nationally representative samples of African-American, European-American, and Mexican-American children and adolescents. The Journal of pediatrics, v. 145, n. 4, p. 439-444, 2004. 16. Slaughter MH, Lohman TG, Boileau RA, Horswill CA, Stillman RJ, Van Loan MD, Bemben DA. Skinfold equations for estimation of body fatness in children and youth. Hum Biol. 1988; 60:709-723.

17. Lohman TG. The use of skinfold to estimate body fatness on children and youth. JOPERD, 1987; 58:98-102.

18. Schaefer L, Plotnikoff RC, Majumdar SR, Mollard R, Woo M, Sadman R et al. Outdoor time is associated with physical activity, sedentary time, and cardiorespiratory fitness in youth. J Pediatr. 2014;165(3):516-21.

19. Hoehner CM, Allen P, Barlow CE, Marx CM, Brownson RC, Schootman M, et al. Understanding the independent and joint associations of the home and workplace built environments on cardiorespiratory fitness and body mass index. Am J Epidemiol. $2013 ; 178(7): 1094-105$.

20. Lo KY, Wu MC, Tung SC, Hsieh CC, Yao HH, Ho CC. Association of School Environment and After-School Physical Activity with Health-Related Physical Fitness among Junior High School Students in Taiwan. Int J Environ Res Public Health. 2017; 14(1): 83.

21. Morgan Hughey S, Kaczynski AT, Child S, Moore JB, Porter D, Hibbert J. Green and lean: is neighborhood park and playground availability associated with youth obesity? variations by gender, socioeconomic status, and race/ethnicity. Am J Prev Med. 2016; S95: S101-S108.

22. Hsieh S, Klassen AC, Curriero FC, Caulfield LE, Cheskin LJ, Davis JN, et al. Built environment associations with adiposity parameters among overweight and obese Hispanic youth. Prev Med Rep. 2015; 2: 406-412. 
23. Albrecht SS, Osypuk TL, Kandula NR, Gallo LC, Lê-Scherban F, Shrager S, et al. Change in waist circumference with longer time in the United States among Hispanic and Chinese immigrants: the modifying role of the neighborhood built environment. Ann Epidemiol. 2015; 25(10): 767-772.

24. Noonan RJ, Boddy LM, Knowles ZR, Fairclough SJ. Cross-sectional associations between high-deprivation home and neighbourhood environments, and health-related variables among Liverpool children. BMJ open. 2016; 6(1): e008693.

25. Carson V, Rosu A, Janssen I. A cross-sectional study of the environment, physical activity, and screen time among young children and their parents. BMC public health, 2014; 14(1): 61.

26. Demment $\mathrm{M}$, Wells $\mathrm{N}$, Olson $\mathrm{C}$. Rural middle school nutrition and physical activity environments and the change in body mass index during adolescence. J Sch Health, 2015;85(2): 100-108.

27. Bürgi R, Tomatis L, Murer K, Bruin ED. Spatial physical activity patterns among primary school children living in neighbourhoods of varying socioeconomic status: a cross-sectional study using accelerometry and Global Positioning System. BMC public health. 2016; 16(1): 282.

28. Tremblay MS, Barnes JD, González SA, Katzmarzyk PT, Onywera VO, Reilly JJ, et al. Global Matrix 2.0: report card grades on the physical activity of children and youth comparing 38 countries. J Phys Act Health, 2016;13(11 Suppl 2): 343-366.

29. Eyre EL, Duncan MJ, Birch SL, Cox V, et al. Environmental and school influences on physical activity in South Asian children from low socio-economic backgrounds: a qualitative study. J Child Health Care. 2015; 19(3): 345-358.

30. Timperio A, Veitch J, Carver A. Safety in numbers: does perceived safety mediate associations between the neighborhood social environment and physical activity among women living in disadvantaged neighborhoods? Am J Prev Med. 2015;74: 49-54.

31. Draper C, Basset S, de Villiers A, Lambert EV; HAKSA Writing Group. Results from South Africa's 2014 report card on physical activity for children and youth. J Phys Act Health. 2014; 11( s1)98-104.

32. Gilliland JA, Rangel CY, Healy MA, Tucker P, Loebach JE, Hess PM, et al. Linking childhood obesity to the built environment: a multi-level analysis of home and school neighbourhood factors associated with body mass index. Can J Public Health. 2012; 103(9): 15-21.

33. Peyer K, Welk GJ, Bailey-Davis L, Chen S. Relationships between County Health Rankings and child overweight and obesity prevalence: a serial cross-sectional analysis. BMC public health. $2016 ; 16(1): 404$.

34. Pereira S, Borges A, Gomes TN, Santos D, Souza M, Dos Santos FK, et al. Correlates of children's compliance with moderate-to-vigorous physical activity recommendations: a multilevel analysis. Scand J Med Sci Sports, 2016.

\section{Willian Giovani de Araújo}

Endereço para correspondência - Universidade de Santa Cruz do Sul, Departamento de Educação Física e Saúde, Curso de Educação Física. Av. Independência, nº 2293

CEP: 96815900 - Santa Cruz do Sul, RS, Brasil.

E-mail: wil.sunshow@gmail.com

Lattes: http://lattes.cnpq.br/3730905345032592

Silvana Silveira Soares - silvanasilveiras@hotmail.com

Ana Paula Sehn - ana_psehn@hotmail.com

Deise Graziela Kern - deisekern@hotmail.com

Letícia Welser - leticia.welser@bol.com.br

Cristiane Fernanda da Silva - cris.cfs@hotmail.com

Miria Suzana Burgos - mburgos@unisc.br

Cézane Priscila Reuter - cezanereuter@unisc.br

\section{Enviado em 23 de março de 2017. Aceito em 13 de junho de 2017.}

